\title{
Dense plasma diagnostics with an amplitude-division soft-x-ray laser interferometer based on diffraction gratings
}

\author{
J. Filevich, ${ }^{*}$ K. Kanizay, M. C. Marconi, ${ }^{*}$ J. L. A. Chilla, and J. J. Rocca \\ Department of Electrical and Computer Engineering, Colorado State University, Fort Collins, Colorado 80523
}

Received November 2, 1999

\begin{abstract}
We report the demonstration of an amplitude-division soft-x-ray interferometer that can be used to generate high-contrast interferograms at the wavelength of any of the saturated soft-x-ray lasers (5.6-46.9 $\mathrm{nm}$ ) that are available at present. The interferometer, which utilizes grazing-incidence diffraction gratings as beam splitters in a modified Mach-Zehnder configuration, was used in combination with a tabletop 46.9-nm laser to probe a large-scale ( 2.7-mm-long) laser-created plasma. () 2000 Optical Society of America

OCIS codes: $340.7450,140.7240$.
\end{abstract}

Interferometry with visible and ultraviolet lasers has been used for more than three decades to obtain multidimensional maps of the electron density in a large variety of dense plasmas. ${ }^{1,2}$ However, free-free absorption and refraction of the probe beam limit the maximum electron density, plasma size, and plasma density gradient that can be probed with optical lasers. $^{3} \quad$ The development of gain-saturated soft-x-ray lasers has opened the possibility of extending laser interferometry to a significantly broader range of plasma parameters. In pioneering interferometry work conducted at Lawrence Livermore National Laboratory, a 15.5-nm Ne-like Y laser pumped by the Nova laser has successfully probed dense laser-created plasmas. ${ }^{3,4}$ The resent advent of gain-saturated tabletop soft-x-ray lasers has created the opportunity to develop portable soft-x-ray tools that will allow detailed maps of the electron-density evolution in a great variety of dense plasmas.

Recently, our group demonstrated plasma interferometry with a tabletop soft-x-ray laser combining a 46.9 -nm capillary-discharge laser with a wave-frontdivision interferometer based on the Lloyd's mirror. ${ }^{5,6}$ The Lloyd's mirror has the advantage of constituting the simplest possible soft-x-ray interferometer. However, it also presents several limitations. First, the probe and the reference beams are very closely spaced. This limits the types of plasma that can be probed. Second, the maximum size of the plasma region that can be probed and the interfringe spacing are not independent, as both depend on the grazing-incidence angle of the Lloyd's mirror. Finally, since this is a wave-front-division interferometer in which the fringe visibility relays the spatial coherence of the laser, it is difficult to maintain good fringe visibility over a large area. This last limitation also applies to Fresnel bimirror interferometers. ${ }^{7}$ These limitations can be overcome by use of an amplitude-division interferometer scheme in which the interference pattern is obtained by superposition of two beams with identical transverse coherence characteristics, such as the skewed Mach-Zehnder interferometer developed by Da Silva et al. that uses thin strained multilayer films to split and recombine the beam. ${ }^{3,4}$ However, this solution cannot be implemented at all wavelengths (e.g., $46.9 \mathrm{~nm}$, the wavelength of our Ne-like Ar laser), owing to the lack of materials with adequate optical constants for the manufacturing of multilayer beam splitters in some regions of the spectrum. To overcome this problem we have developed a novel soft-x-ray amplitude-division interferometer in which diffraction gratings are used as beam splitters. By proper tailoring of the gratings, the interferometer can be made to operate at any selected soft-x-ray wavelength. An additional advantage of the use of diffraction gratings is their increased resistance to catastrophic damage produced by fast particle debris. A phase-shifting point diffraction soft-x-ray interferometer that uses a grating to improve the efficiency was recently demonstrated by Medecki et al. ${ }^{8}$

The soft-x-ray interferometer, which consists of a modified Mach-Zehnder configuration, ${ }^{9}$ is schematically illustrated in Fig. 1. The soft-x-ray laser beam

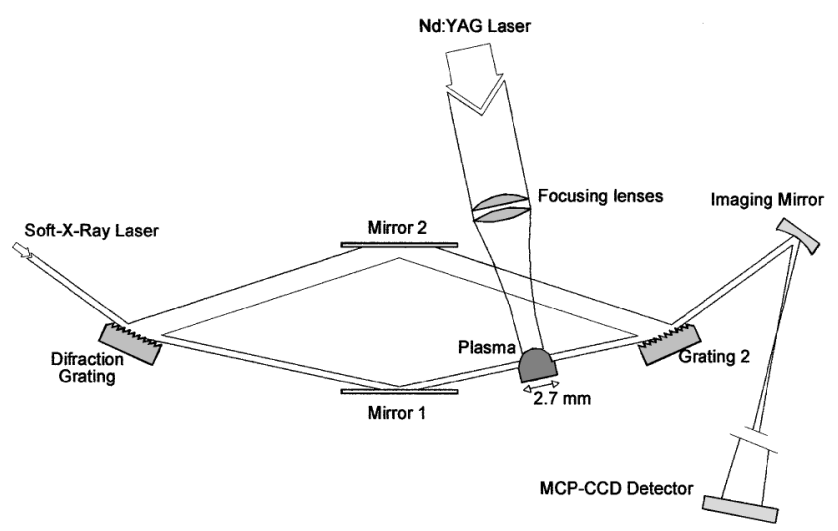

Fig. 1. Schematic diagram of the soft-x-ray amplitudedivision grating interferometer and plasma diagnostics setup. 
is diffracted off the first grating into the two arms of the interferometer. The incidence angle and the blaze of the grating are selected to allow for most of the laser radiation to be evenly split between the zero and the first diffraction orders. This even split is essential for obtaining good fringe visibility. Elongated grazingincidence mirrors are placed on the zero- and the firstorder arms to redirect the light toward the second grating, which recombines the beams to generate the interference pattern. We can adjust the fringe spacing and the orientation by changing the tilt on the grazingincidence mirrors. The plasma that is to be studied is introduced into one of the arms of the interferometer. $\mathrm{A} \mathrm{Si} / \mathrm{Sc}$ multilayer mirror ${ }^{10}$ of $30-\mathrm{cm}$ focal length and $\approx 40 \%$ reflectivity images the plasma with a magnification of $\sim 25 \times$ onto a microchannel plate (MCP) CCD detector. A flat mirror (not shown in Fig. 1) was used to relay the image.

The interferometer was prealigned with an infrared (824-nm) semiconductor laser diode selected to have a temporal coherence length of $\sim 250 \mu \mathrm{m}$, similar to that of the soft-x-ray laser. To allow the alignment of the interferometer with the diode laser we designed the gratings with two vertically separated rulings on the same substrate. We ruled one part of the substrate with 300 lines $/ \mathrm{mm}$ to diffract the $46.9-\mathrm{nm}$ laser beam and the other with 17.06 lines $/ \mathrm{mm}$ to diffract the beam of the alignment diode. With this ratio of groove spacing we obtained the same dispersion and therefore the same path for the beams of the soft-x-ray laser and the alignment laser diode. We motorized all the relevant movements in the system to fine tune the interferometer once the system was in vacuum. Interferograms with very good visibility $(\sim 0.5)$ were obtained over the entire field of view. The interferometer showed excellent stability.

The interferometer was used in combination with a 46.9-nm capillary-discharge tabletop laser ${ }^{11}$ to map the electron-density evolution of a line-focused laser-created plasma generated on a $\mathrm{Cu}$ slab target. We generated the plasma by focusing a $Q$-switched $\mathrm{Nd}$ :YAG laser operating at its fundamental wavelength of $1.06 \mu \mathrm{m}$ into an $\sim 30-\mu \mathrm{m}$-wide line focus $\sim 2.7 \mathrm{~mm}$ in length. The results discussed below correspond to the study of a plasma created with 0.36-J Nd:YAG laser pulses of $\sim 13$-ns FWHM duration. The probe-laser pulses were generated by amplification of the 46.9-nm line of $\mathrm{Ne}$-like $\mathrm{Ar}$ in an Ar capillary plasma excited by a fast-discharge current pulse. This tabletop laser produces pulses with an energy of $\sim 0.13 \mathrm{~mJ}$ and 1.2-ns FWHM duration. ${ }^{11}$ The far-field beam profile has an annular shape and a peak-to-peak divergence of $\sim 4.6 \mathrm{mrad}$.

Figure 2 shows a sequence of interferograms that describe the evolution of the laser-created plasma. The fringes are observed to shift with time owing to the presence of the plasma. At the later times the plasma cools, and absorption of the probe beam by the plasma becomes noticeable. Electron-density maps were computed from the measured number of fringe shifts at each location, assuming that the plasma is uniform along the line focus. Figure 3 illustrates a sequence of two-dimensional electrondensity distributions corresponding to the sequence of interferograms shown in Fig. 2. The formation, expansion, and decay of the plasma at the end of the pump-laser pulse can be observed. We have been able to probe the plasma at locations as close as $25-30 \mu \mathrm{m}$ from the target, where the electron density is $\approx 5 \times 10^{19} \mathrm{~cm}^{-3}$ and the density gradient is steep. Another series of measurements for $1.8-\mathrm{mm}$ long line-focused plasmas created by 0.6-J Nd:YAG laser pulses probed electron densities as great as $1 \times 10^{20} \mathrm{~cm}^{-3}$. Ray-tracing computations show that these measurements would be difficult to realize with an ultraviolet laser, owing to strong refraction caused by the large density gradients. This is illustrated in Fig. 4, which shows that, although refraction of the soft-x-ray laser probe is small, a hypothetical 265-nm probe beam would be totally deflected out of the plasma.

In conclusion, we have demonstrated a new soft-xray interferometer that one can combine with any of the currently available saturated soft-x-ray lasers to study many high-density laboratory plasmas that are of significant interest. The use of this interferometer with

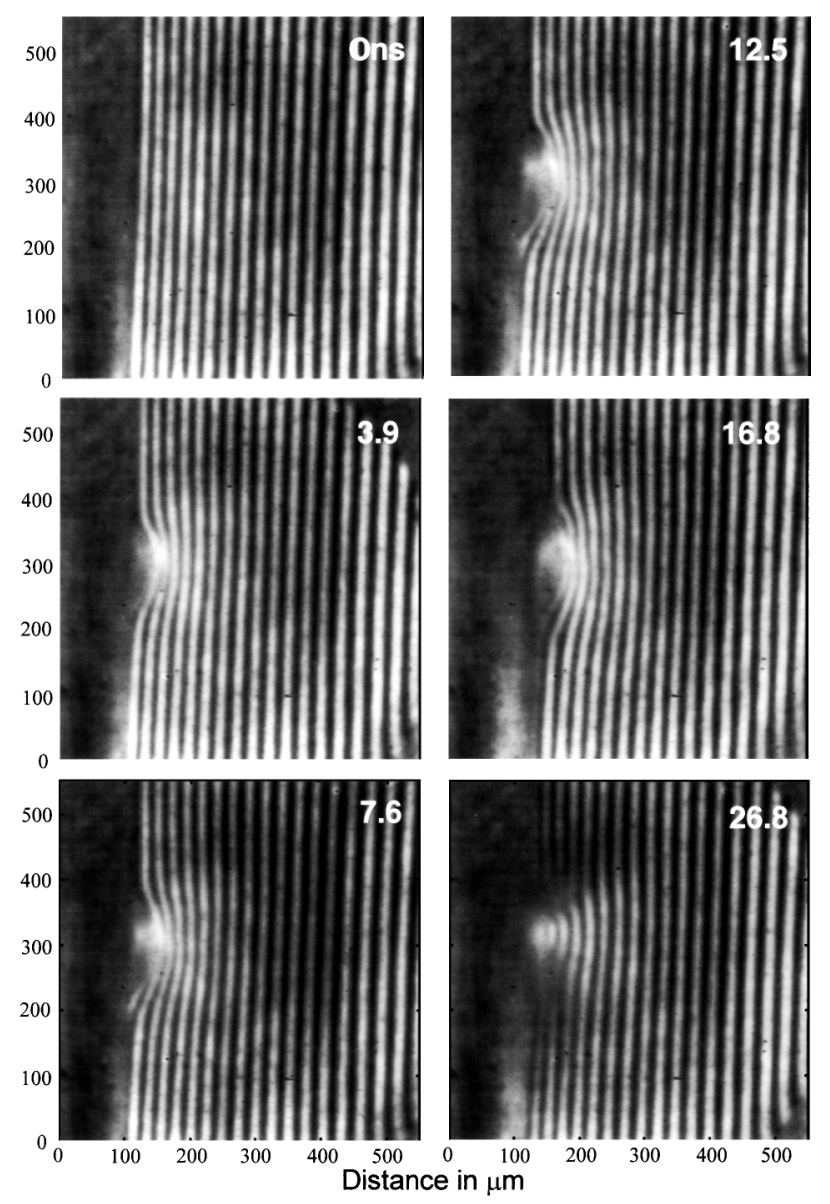

Fig. 2. Sequence of soft-x-ray interferograms depicting the evolution of a 2.7-mm-long line-focused plasma generated by a Nd:YAG laser $(\lambda=1.06 \mu \mathrm{m}, 0.36 \mathrm{~mJ}, 13 \mathrm{~ns}$, FWHM). The time delay with respect to the beginning of the Nd:YAG laser pulse is shown in each interferogram. 

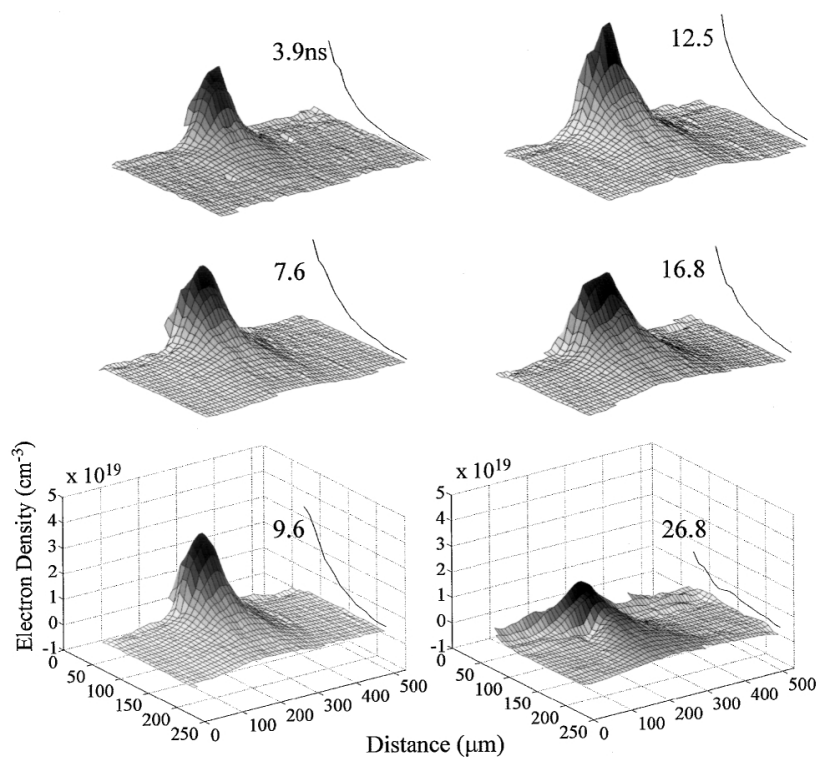

Fig. 3. Electron-density distributions corresponding to the interferograms of Fig. 2.

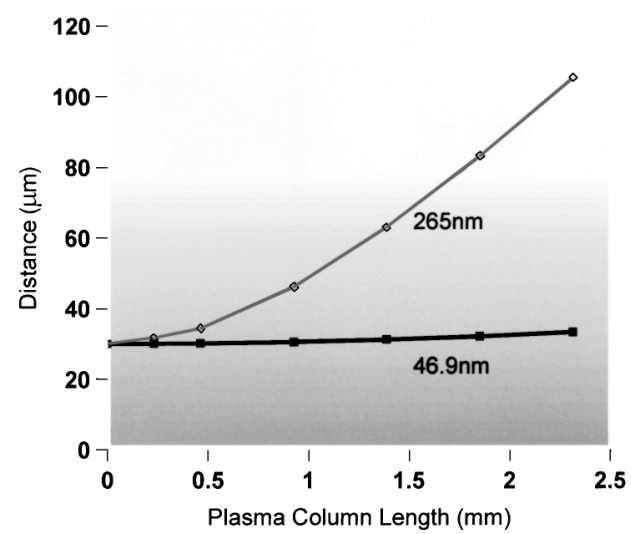

Fig. 4. Refraction of 46.9- and 265-nm probe beams propagating through a plasma with the density gradient corresponding to the electron-density distribution measured $12.5 \mathrm{~ns}$ after initiation of the laser pulse. The probe beams are assumed to enter the plasma parallel to the target at a distance of $30 \mu \mathrm{m}$ from the surface.

shorter-wavelength soft-x-ray lasers of short pulse duration will allow the probing of near-solid-density plasmas.
This work was supported by U.S. Department of Energy grant DE-FG03-98DP00208. The development of the soft-x-ray laser was supported by the National Science Foundation. M. Marconi acknowledges the support of the Universidad de Buenos Aires and the Consejo Nacional de Investigaciones Científicas y Técnicas. We thank R. J. Berklund and O. E. Martinez for their contributions and A. Vinogradov, Yu. Unspenskii, and their groups for the development of the multilayer mirrors. J. J. Rocca's e-mail address is rocca@engr.colostate.edu.

*Permanent address, Departamento de Fisica, Facultad de Ciencias Exactas y Naturales, Universidad de Buenos Aries, Buenos Aires, Argentina.

\section{References}

1. D. T. Attwood, D. W. Sweeney, J. M. Auerbach, and P. H. Y. Lee, Phys. Rev. Lett. 40, 184 (1978).

2. M. D. Rosen, P. L. Hagelstein, D. L. Matthews, E. M. Campbell, A. U. Hazi, B. L. Whitten, B. MacGowan, R. E. Turner, R. W. Lee, G. Charatis, G. E. Bush, C. L. Shepard, and P. D. Rockett, Phys. Rev. Lett. 54, 106 (1985).

3. L. B. Da Silva, T. W. Barbee, Jr., R. Cauble, P. Celliers, D. Ciarlo, S. Libby, R. A. London, D. Matthews, S. Mrowka, J. C. Moreno, D. Ress, J. E. Trebes, A. S. Wan, and F. Weber, Phys. Rev. Lett. 74, 3991 (1995).

4. A. S. Wan, T. W. Barbee, Jr., R. Cauble, P. Celliers, L. B. Da Silva, J. C. Moreno, P. W. Rambo, G. F. Stone, J. E. Trebes, and F. Weber, Phys. Rev. E 55, 6293 (1997).

5. J. J. Rocca, C. H. Moreno, M. C. Marconi, and K. Kanizay, Opt. Lett. 24, 420 (1999).

6. C. H. Moreno, M. C. Marconi, K. Kanizay, J. J. Rocca, Yu. A. Unspenskii, A. V. Vinogradov, and Yu. A. Pershin, Phys. Rev. E 60, 911 (1999).

7. F. Albert, D. Joyeaux, P. Jaeglé, A. Carillon, J. P. Chauvineau, G. Jamelot, A. Klishnick, J. C. Lagron, D. Phalippou, D. Ros, S. Sebban, and P. Zeitoun, Opt. Lett. 142, 184 (1997).

8. H. Medecki, E. Tejnil, K. A. Goldberg, and J. Bokor, Opt. Lett. 21, 1526 (1996).

9. J. L. A. Chilla, J. J. Rocca, O. E. Martinez, and M. C. Marconi, Opt. Lett. 21, 955 (1996).

10. Yu. A. Unspenskii, V. A. Lavashov, A. V. Vinogradov, A. I. Fedorenko, V. V. Kondratenko, Yu. P. Pershin, E. N. Zubarev, and V. Yu Fedotov, Opt. Lett. 23, 771 (1998).

11. B. R. Benware, C. D. Macchietto, C. H. Moreno, and J. J. Rocca, Phys. Rev. Lett. 81, 5804 (1998). 